\title{
Feasibility and Safety of Multicenter Tissue and Biofluid Sampling for $\alpha$-Synuclein in Parkinson's Disease: The Systemic Synuclein Sampling Study (S4)
}

Lana M. Chahine ${ }^{\mathrm{a}, *}$, Thomas G. Beach ${ }^{\mathrm{b}}$, Nicholas Seedorff ${ }^{\mathrm{c}}$, Chelsea Caspell-Garcia ${ }^{\mathrm{c}}$, Christopher S. Coffey ${ }^{c}$, Michael Brumm ${ }^{c}$, Charles H. Adler ${ }^{d}$, Geidy E. Serrano ${ }^{b}$, Carly Linder ${ }^{\mathrm{e}}$, Sherri Mosovsky ${ }^{\mathrm{a}}$, Tatiana Foroud ${ }^{\mathrm{f}}$, Holly Riss ${ }^{\mathrm{c}}$, Dixie Ecklund ${ }^{\mathrm{c}}$, John Seibyl ${ }^{\mathrm{g}}$, Danna Jennings ${ }^{\mathrm{h}}$, Vanessa Arnedo ${ }^{i}$, Lindsey Riley ${ }^{i}$, K.D. Dave ${ }^{i}$ and Brit Mollenhauer ${ }^{j}$ on behalf of the Systemic Synuclein Sampling study ${ }^{1}$

a Department of Neurology, University of Pittsburgh, Pittsburgh, PA, USA

${ }^{\mathrm{b}}$ Civin Laboratory for Neuropathology, Banner Sun Health Research Institute, Sun City, AZ, USA

${ }^{\mathrm{c}}$ Department of Biostatistics, The University of Iowa, Iowa City, IA, USA

${ }^{\mathrm{d}}$ Department of Neurology, Mayo Clinic, Scottsdale, AZ, USA

${ }^{\mathrm{e}}$ Department of Neurology, University of Pennsylvania Perelman School of Medicine, Philadelphia, PA, USA

${ }^{\mathrm{f}}$ Department of Medical and Molecular Genetics, Indiana University School of Medicine, Indianapolis, IN, USA

${ }^{\mathrm{g}}$ Institute for Neurodegenerative Disorders, New Haven, CT, USA

${ }^{\mathrm{h}}$ Denali Therapeutics, San Francisco, CA, USA

${ }^{i}$ The Michael J. Fox Foundation for Parkinson's Research, New York, NY, USA

${ }^{\mathrm{j}}$ Paracelsus-Elena-Klinik, Kassel and University Medical Center Goettingen, Goettingen, Germany

Accepted 20 August 2018

\begin{abstract}
.
Background: $\alpha$-synuclein is a lead Parkinson's disease (PD) biomarker. There are conflicting reports regarding accuracy of $\alpha$-synuclein in different tissues and biofluids as a PD biomarker, and the within-subject anatomical distribution of $\alpha$-synuclein is not well described. The Systemic Synuclein Sampling Study (S4) aims to address these gaps in knowledge. The S4 is a multicenter, cross-sectional, observational study evaluating $\alpha$-synuclein in multiple tissues and biofluids in PD and healthy controls (HC).

Objective: To describe the baseline characteristics of the S4 cohort and safety and feasibility of this study.

Methods: Participants underwent motor and non-motor clinical assessments, dopamine transporter SPECT, biofluid collection (cerebrospinal fluid, saliva, and blood), and tissue biopsies (skin, sigmoid colon, and submandibular gland). Biopsy adequacy was determined based on presence of adequate target tissue. Tissue sections were stained with the 5C12 monoclonal antibody against unmodified $\alpha$-synuclein. All specimens were acquired and processed in a standardized manner. Adverse events were systematically recorded.
\end{abstract}

\footnotetext{
${ }^{1}$ See appendix.

${ }^{*}$ Correspondence to: Lana M. Chahine, 3471 Fifth Avenue, Suite 811, Pittsburgh, PA 15213, USA. Tel.: +1 412692 4916; Fax: +1 412692 4601; E-mail: lchahine2018@gmail.com.
} 
Results: The final cohort consists of 82 participants (61 PD, $21 \mathrm{HC}$ ). In 68 subjects (83\%), all types of specimens were obtained but only $50(61 \%)$ of subjects had all specimens both collected and evaluable for $\alpha$-synuclein. Mild adverse events were common, especially for submandibular gland biopsy, but only 1 severe adverse event occurred.

Conclusion: Multicenter tissue and biofluid sampling for $\alpha$-synuclein is feasible and generally safe. $\mathrm{S} 4$ will inform understanding of the concurrent distribution of $\alpha$-synuclein pathology and biomarkers in biofluids and peripheral nervous system in PD.

Keywords: Biomarkers, needle biopsy, Parkinson's disease, synucleins

\section{INTRODUCTION}

A major obstacle to development of Parkinson's disease (PD) therapeutics is the lack of objective disease measures. Abnormal accumulation of $\alpha$-synuclein in the brain is the main pathological hallmark in PD and also shows a gene dosage effect [1], making it a lead candidate PD biomarker. Multiple reports cite $\alpha$-synuclein changes in different biofluids and tissues in PD [2] and prodromal PD [3]. However, there are conflicting reports regarding sensitivity and specificity of $\alpha$-synuclein in biofluids and different tissues as a PD biomarker. The explanation for these conflicting reports is likely multifactorial including small sample sizes, generally from single centers without formal study monitoring, as well as differences in study sample, acquisition, processing, tissue staining, and interpretation [4]. Furthermore, it is unknown how levels of $\alpha$-synuclein correlate in different matrices (tissue and biofluids) in the same individual. Better understanding of $\alpha$-synuclein distribution in different tissues and body fluids is essential to identifying measures of $\alpha$-synuclein that serve as accurate biomarkers for diagnosis, disease progression, target engagement and therapeutic efficacy. To fully assess the utility of a biomarker for these purposes, it is essential to account for the feasibility of a biomarker's widespread adoption and dissemination, including ease and safety of specimen acquisition.

The Systemic Synuclein Sampling Study (S4) is a multicenter study that aimed to collect multiple tissues and biofluids within the same subject, using rigorous data/specimen collection and storage as well as standard protocols at each step from acquisition to interpretation. Subject recruitment and specimen acquisition have been completed. We herein report on the baseline characteristics of participants in the S4 study, as well as the feasibility and safety of obtaining multiple tissue and biofluid specimens within the same subjects.

\section{METHODS}

\section{Study sample}

S4 is a cross-sectional study evaluating $\alpha$ synuclein pathology in multiple tissues and biofluids in the same PD and HC subjects. Study methodology have been described in detail elsewhere [5]. Briefly, between October 2015 and August 2017, the study aimed to enroll individuals with PD belonging to one of 3 groups: 20 early PD patients within 2 years of diagnosis, not treated with dopaminergic therapy; 20 patients with moderate disease, 2-5 years duration, without motor fluctuations; and 20 advanced PD with disease duration of 5 or more years and with motor fluctuations and/or dyskinesias. Decreased dopamine transporter (DaT) SPECT binding, based on age- and sex- matched normative data, was required for inclusion [6]. In addition, a non-PD healthy comparator (HC) group, with normal DaT SPECT, was recruited. Exclusion criteria in both $\mathrm{PD}$ and $\mathrm{HC}$ groups included clinical diagnosis of dementia (Montreal Cognitive Assessment (MoCA) score was accounted for in determining eligibility of potential HC but not PD participants), diabetes, bleeding diathesis, history of submandibular gland botulinum toxin injections for sialorrhea, active gastroenterologic-, dermatologic-, or salivary gland disease/disorder and/or any conditions that preclude the safe performance of the lumbar puncture, flexible sigmoidoscopy, skin biopsy, or submandibular gland biopsy. While the goal was to obtain all biofluids and all tissues on all subjects, a minimum of 2 biofluid types and 2 tissue procedures were required to consider subjects as having completed study participation; target recruitment was 80 subjects with at least 2 biofluids and 2 tissues collected.

The study was approved by institutional review boards at each S4 site, and written informed consent was obtained. 


\section{Tissue and biofluid acquisition procedures}

Biopsies of the skin, sigmoid colon, and submandibular gland were obtained as described [5] and are detailed in the S4 biologics manual [7]. Briefly, skin biopsies were performed using a $3 \mathrm{~mm}$ punch biopsy tool under local anesthesia. This procedure was performed by the site PI (Neurologist) at 3 sites, by a PA at 2 sites, and a surgeon at 1 site. Two biopsies were obtained from the paravertebral posterior cervical region and 2 from the ipsilateral mid-thigh. Colon biopsy was performed by gastroenterologists through flexible sigmoidoscopy. Up to 8 biopsies were obtained. Submandibular gland needle biopsy was performed by otolaryngologists under local anesthesia using a 16-gauge core biopsy instrument. Initially, up to 5 biopsies were obtained, but after the occurrence of a serious adverse event (see below) 12 months into the study, the protocol was changed to allow up to 4 biopsies. Ultrasound guidance of submandibular gland biopsy was optional and was used at only 1 of the 6 study sites. Proceduralist training was performed by a specialist experienced in each biopsy type (experienced generally, and specifically in individuals with PD). Training occurred via live webinar.

Biofluids were obtained and processed as previously described [5] and are detailed in the S4 biologics manual [7]. Briefly, lumbar puncture (LP) was performed with a goal volume of collection of $20 \mathrm{~mL}$ of cerebrospinal fluid (CSF). Use of a 24 gauge (g) atraumatic (Sprotte) needle was recommended, but $20 \mathrm{~g}$ or $22 \mathrm{~g}$ needles were allowed in instances where fluoroscopy-guided LP was performed. Passive saliva collection was performed, with a goal of $5 \mathrm{~mL}$ collected. Blood was collected by venous puncture and processed into plasma, serum, and whole blood.

The goal was for acquisition of all specimens to occur within 120 days of the screening visit.

\section{Specimen processing and staining/assaying for $\alpha$-synuclein}

The flow of specimens and determination of specimen adequacy at each step is shown schematically in Fig. 1.

Tissue processing and packaging steps have been previously described [5] and are detailed in the S4 biologics manual [7]. Briefly, skin, submandibular gland, and colon tissue biopsy specimens were placed between sponges within a standard plastic embedding cassette and then immersed in 10\% formalin (2-3 biopsy fragments were placed in each cassette). These were then shipped to the biorepository where all contents of a single cassette were embedded into a paraffin block. Each formalin-fixed paraffinembedded (FFPE) block was cut completely through into $4 \mu \mathrm{m}$ sections mounted on individual slides. Four equidistant sections from each cassette were stained with hematoxylin and eosin (H\&E). Images of the $H \& E$ stained slides were sent to the neuropathology core for determination of presence of adequate target tissue (see below). Three slides from each cassette with adequate target tissue were selected and shipped to a contract research organization (CRO) Targos Molecular Pathology GmbH, located in Kassel, Germany. There, paraffin-embedded tissue sections were stained on an automated Ventana BenchMark ${ }^{\circledR}$ platform with the 5C12 monoclonal antibody as described [8]. Positive and negative control slides were included with each staining run. These consisted of slides of post-mortem neuropathologically examined tissue ( 2 each of cerebral cortex, skin (posterior scalp), and colon) obtained from PD and HC subjects known to be clearly positive or negative for $\alpha$-synuclein pathology respectively. Once positive and negative controls were reviewed, digital images of the slides were then taken at $40 \mathrm{x}$ resolution, placed on flash drives, and shipped to the neuropathology core (core PI author TGB, at Banner Sun Health Research Institute) as well as to 3 independent and blinded histopathology judges (medically-certified neuropathologists). The neuropathology core and histopathology judges individually reviewed and rated the slides semi-quantitatively for presence of $\alpha$-synuclein.

Biofluids were processed as detailed in the S4 biologics manual [7]. Briefly, CSF was centrifuged at $2000 \mathrm{xg}$ for 10 minutes at room temperature $\left(18^{\circ} \mathrm{C}\right.$ to $\left.25^{\circ} \mathrm{C}\right)$. The supernatant was aliquoted into pre-cooled polypropylene CSF collection aliquot tubes, placed on dry ice within 60 minutes of collection, and then stored at $-80^{\circ} \mathrm{C}$ until shipped on dry ice to the biorepository. As for saliva, $10 \mathrm{X}$ protease inhibitor cocktail (PIC) was added to the saliva sample and, within 30 minutes of collection, centrifuged at $4^{\circ} \mathrm{C}$ at $2000 \mathrm{xg}$ for 15 minutes. Saliva was then aliquoted into pre-cooled microcentrifuge tubes and stored at $-80^{\circ} \mathrm{C}$ until shipped on dry ice to the biorepository. Blood was collected by venous puncture into EDTA tubes for whole blood, DNA, plasma, and buffy coat. Blood was also collected in a serum tube for serum aliquots and PAXGene 


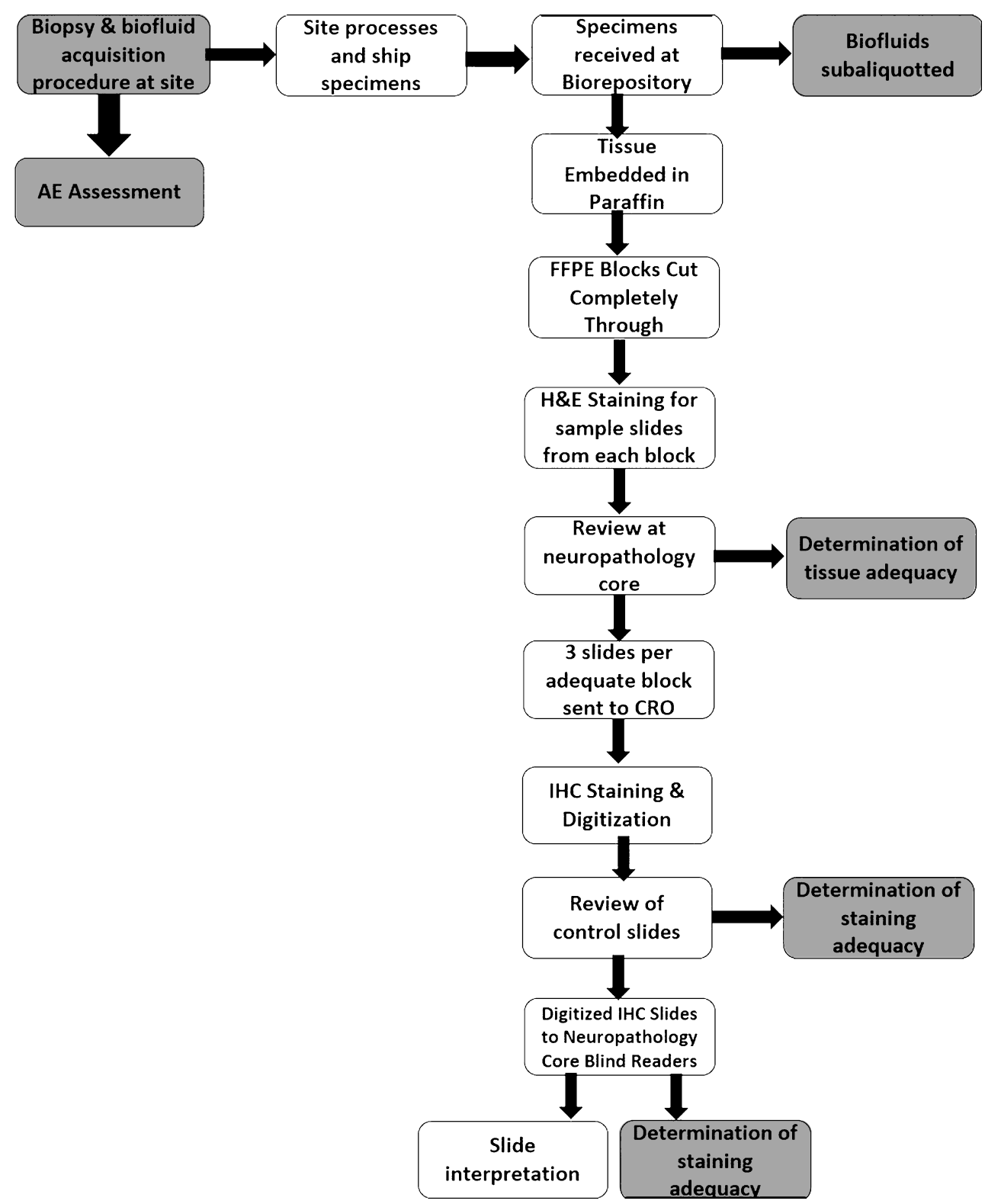

Fig. 1. Steps in tissue processing and assessment of adequacy in S4. Steps for assessments of safety and feasibility are highlighted in grey. $\mathrm{AE}$, adverse event; $\mathrm{CRO}$, contract research organization; FFPE, formalin-fixed paraffin-embedded; H\&E, hematoxylin and eosin.

tubes for RNA. Specimens were processed as detailed in the $\mathrm{S} 4$ biologics manual [7] and stored at $-80^{\circ} \mathrm{C}$ until shipped on dry ice to the biorepository. DNA and RNA were extracted as described (http://www.ppmi-info.org/study-design/researchdocuments-and-sops/), with one modification: in S4, one RNA tube was utilized for miRNA extraction and another for total RNA extraction, with 2 remaining PAXgene tubes stored for future use.
Total $\alpha$-synuclein was measured on CSF, saliva, whole blood, plasma, and serum using enzymelinked immunosorbent assay (ELISA) BioLegend $\alpha$-Synuclein Immunoassay (BioLegend, San Diego, CA, USA; Cat. No. 844101) as described [9]. Concentrations of hemoglobin in plasma and serum were measured using the QuantiChrom test (BioAssay Systems, Cat. No. DIHB-250)). Concentration of hemoglobin in CSF was measured using the 
Human Hemoglobin ELISA Quantitaton Set (Bethyl Laboratories, Cat. No. E88-134). Concentrations of hemoglobin in whole blood and saliva were measured using the enzyme-linked immunosorbent assay from Bethyl Laboratories (Cat. No. E88-134).

\section{Metrics of feasibility}

Feasibility of the tissue acquisition procedure was captured at various stages of the study, as depicted in the schematic shown in Fig. 1. Feasibility was quantified using several metrics:

1) Whether the procedure was attempted and whether any tissue was obtained, as documented by the study coordinator on the case report form.

2) Whether the tissue obtained contained "adequate" target tissue, as determined following review of the H\&E stained slides at the neuropathology core. Adequate biopsy was defined by at least one fragment of a given tissue type containing a minimal amount $\left(2 \mathrm{~mm}^{2}\right)$ of target tissue. Target tissue was defined as glandular tissue (epithelial secretory acini and intervening stroma) for submandibular gland, submucosa for colon, and dermis for skin. Multiple cassettes (each containing one or more fragments of biopsy) could be obtained per subject per tissue biopsy. A binary determination of adequate biopsy, defined as a minimum of one cassette with adequate target tissue for a given tissue type for a given subject, was assessed. An inadequate specimen was defined by absence or insufficient amount of target tissue in a given cassette.

3) Feasibility of staining was assessed by the number of slides that were determined to have been adequately stained divided by the total number of stained slides. This determination was jointly made for each slide by the CRO and the neuropathology core.

Biofluid collection feasibility was assessed with number of specimens and volume collected. In addition, CSF samples with hemoglobin level of $>200 \mathrm{ng} / \mathrm{mL}$ were also considered as a metric of feasibility, as this degree of hemoglobin contamination of CSF may be significant enough to affect measurements of CSF total $\alpha$-synuclein [10].

\section{Metrics of safety}

Adverse event data were collected at the visits (during in-person subject assessment by the site coordinator and/or PI) and during follow-up via phone calls performed at 7 days ( \pm 2 days) of each procedure. Adverse events (AEs) were graded 1-5 as defined in the Common Terminology Criteria for Adverse Events (CTCAE) [11], and summarized as follows: mild, grade 1: aware of event but easily tolerated; moderate, grade 2: discomfort, enough to cause interference with usual activity; severe, grade 3: incapacitating: subject unable to work or perform usual activities; life-threatening, grade 4; and death, grade 5. A serious adverse event (SAE) was defined as a grade 4 or 5 AE. Only AE's considered to be at least possibly related to a study procedure are considered in this analysis.

\section{Statistical analysis}

Analyses were performed using SAS 9.4 (SAS Institute Inc., Cary, NC). Descriptive statistics were used to summarize basic demographics and metrics of safety and feasibility. For continuous variables, differences between the PD and HC groups were examined using two-sample $t$-tests or Wilcoxon rank sum tests, where appropriate. For ordinal variables the Cochran-Armitage test for trend was used while chi-squared tests or Fisher's exact tests were implemented for categorical variables.

A range of generalized linear models and generalized linear mixed models were used to explore predictors of the various response variables. A logistic regression model was used to explore the predictors of inadequate tissue acquisition while linear regression models were used to look at associations of saliva volume. Adverse event rate was calculated as the number of AEs per number of procedures assessed. The difference in adverse event rates between groups was evaluated using a negative binomial model (due to evidence of overdispersion).

A mixed effects logistic regression model was used to explore the relationship between study site and the odds of obtaining an inadequate sample. The model included a random intercept that clustered on individual while also adjusting for tissue type.

\section{RESULTS}

Of 100 individuals who participated in S4 screening activities, 18 were deemed ineligible to participate. Reasons for exclusion were: MoCA score $(n=5)$, negative DaT SPECT scan $(n=6)$, antiplatelet/anticoagulant use $(n=1)$, comorbid disease (cancer, diabetes; $n=2$ ), did not qualify based 
on PD disease duration and/or manifestations $(n=2)$, and not suitable for study participation per investigator discretion (not otherwise specified; $n=2$ ).

Eighty-two subjects were enrolled, 61 with PD and $21 \mathrm{HCs}$. Characteristics of enrolled subjects are shown in Table 1 . Of those that enrolled, 80 completed collection of at least 2 biofluids and 2 tissues. Mean time for completion of study participation was 41 days (range 2-127).

Feasibility data are shown in Table 2. Skin biopsy was collected on all but 2 subjects, both of whom declined to undergo the procedure. Colon biopsy was attempted on all but 1 subject who declined to have the study procedure. Submandibular gland biopsy was not attempted in 5 subjects who also declined to have this procedure; in 1 instance submandibular gland biopsy was attempted but tissue could not be obtained. Adequacy of obtained target tissue was high for skin and colon but lower for submandibular gland (among specimens obtained, percent with at least some adequate tissue: $97.6 \%$ for skin, $96.3 \%$ for colon, $68.3 \%$ for submandibular gland). The odds of an inadequate submandibular gland specimen increased by $11 \%$ for each 1 -year increase in age $(\mathrm{OR}=1.11 ; 95 \%$ CI $1.03-1.19 ; p<0.006)$. There were no significant associations between biopsy adequacy and sex, group (PD vs. HC), or PD disease duration or severity (data not shown). The number of subjects with at least 1 adequate submandibular gland specimen obtained was highest at one study site that used ultrasound-guidance [11 (85\%) of 13 enrolled subjects at that site] and one site that did not [9(82\%) of 11 subjects enrolled at that site]. At other sites, all of which did not use ultrasound guidance, the percentage of subjects with at least 1 adequate specimen ranged from $44-74 \%$. The proportion of submandibular specimens obtained that were inadequate was not lower at the site that used ultrasound $(13 \%)$ vs. sites that did not $(10-67 \%)(p=0.55)$. Forty-one $(67 \%)$ of the PD group and 14 (67\%) of the HC group had adequate specimens of each of the 3 tissue types.

In regards to biofluid collection, CSF collection was attempted on all $\mathrm{HC}$ and 60 (98\%) PD subjects and successfully obtained in $75(93 \%)$ of those attempted. Ten CSF samples had a hemoglobin level of $>200 \mathrm{ng} / \mathrm{mL}$. The proportion of specimens with $\mathrm{Hb}>200$ was greater for the $10 \mathrm{CSF}$ specimens obtained with LP needle gauge of 20 or $22(3 / 10$ $(30 \%))$, though this difference was not statistically significant $(p=0.12)$.

Saliva collection was attempted on all subjects but collected saliva volume was lower than goal for many participants. This was anecdotally reported by coordinators to be due to dry mouth/inability of the participant to produce saliva. The volume of saliva collected was not associated with age, sex, disease duration, current or former tobacco use, hours since last food/liquid intake, and hours since last use of oral hygiene products (data not shown). Blood samples were obtained on all but 1 subject.

All possible tissues and biofluids were collected on $48(79 \%)$ of the PD group and $20(95 \%)$ of the HC group ( $p=0.10$ for difference between groups). However, a lesser fraction, 36 (59\%) PD and 14 (67\%) HC subjects, had evaluable specimens of all biofluids and tissue. This difference between the number with all specimens collected vs. all specimens adequate (eg evaluable for $\alpha$-synuclein) is mainly accounted for by the proportion of inadequate specimens obtained with submandibular gland biopsy. If CSF hemoglobin (which could influence measurement of CSF total $\alpha$ synuclein) is accounted for in the evaluability of CSF, 30 (49\%) PD and 13 (62\%) HC subjects had all specimens collected and evaluable (with evaluable here defined as CSF hemoglobin $<200 \mathrm{ng} / \mathrm{dL}$, and at least 1 adequate cassette for each tissue type).

Safety data are shown in Table 3. In regards to adverse events (AEs), among those who had colon biopsy, only $1 \mathrm{AE}$ was reported, documented as diarrhea following the procedure. Eight AEs were reported in those who had skin biopsy, including 3 with contusions, 2 with pain, and two with signs of inflammation. Seventy AEs from submandibular gland were reported. The vast majority of AEs from SMG biopsy were mild/grade I (76\%); $25 \%$ were moderate. The most commonly reported AEs with SMG included pain in 22 instances, swelling in 20 , hematoma and/or contusion in 21. In regards to AEs with biofluid collection, mild AEs were reported for CSF collection in $12 \%$ of those who underwent the procedure (Table 3). There were no AEs reported for saliva collection or phlebotomy. There were no significant differences in AE rate in the PD group vs. the $\mathrm{HC}$ group for SMG biopsy (rate of AE for other tissue type biopsies was too low to compare for significant differences between groups).

There was 1 serious adverse event in the entire study, related to SMG biopsy complicated by sublingual hematoma which manifested with pain, dysarthria, dysphagia and dyspnea. This resolved without intervention.

A total of 106,624 slides were generated from S4 specimens: 35,316 skin, 44,383 colon, and 26,925 submandibular gland. A total of 1,782 were sent to 
Table 1

S4 Cohort demographic and clinical characteristics $\mathrm{SD}=$ standard deviation

\begin{tabular}{|c|c|c|}
\hline Variable & $\mathrm{PD} \mathrm{N}=61$ & $\mathrm{HC} \mathrm{N}=21$ \\
\hline Mean Age in years (SD; range) & $62.9(8.6 ; 43.6-85.5)$ & $61.0(6.3 ; 51.3-71.3)$ \\
\hline \multicolumn{3}{|l|}{ Gender, N (\%) } \\
\hline Male & $41(67)$ & $9(43)$ \\
\hline Female & $20(33)$ & $12(57)$ \\
\hline \multicolumn{3}{|l|}{ Ethnicity, N (\%) } \\
\hline Hispanic/Latino & $1(2)$ & $0(0)$ \\
\hline Not Hispanic/Latino & $60(98)$ & $21(100)$ \\
\hline \multicolumn{3}{|l|}{ Race, N $(\%)$} \\
\hline White & $59(97)$ & $20(95)$ \\
\hline Not White & $2(3)$ & $1(5)$ \\
\hline Mean Years of Education (SD; range) & $17.6(3.09 ; 10.0-25.0)$ & $17.19(3.17 ; 12.0-24.0)$ \\
\hline Mean Symptom Duration (Months) (SD; range) & $74.8(57.2 ; 2.8-251.3)$ & N/A \\
\hline Mean Disease Duration in months (SD; range) & $56.0(54.9 ; 1.1-245.3)$ & N/A \\
\hline Mean MDS-UPDRS Total Off Score (SD; range) & $44.2(19.0 ; 8.0-90.0)$ & $4.3(3.7 ; 0.0-14.0)$ \\
\hline Missing* & 5 & 0 \\
\hline Mean MDS-UPDRS III Off Score (SD; range) & $26.5(11.8 ; 7.0-56.0)$ & $1.1(2.31 ; 0.0-10.0)$ \\
\hline Missing & 5 & 0 \\
\hline \multicolumn{3}{|l|}{ Hoehn and Yahr Stage, N (\%)** } \\
\hline 0 & $1(1.6)$ & N/A \\
\hline 1 & $12(19.7)$ & \\
\hline 2 & $41(67.2)$ & \\
\hline 3 & $7(11.5)$ & \\
\hline
\end{tabular}

Table 2

Feasibility of tissue and biofluid collection. $\mathrm{SD}=$ Standard deviation

\begin{tabular}{|c|c|c|c|c|}
\hline Specimen type & Metric & $\mathrm{PD}(\mathrm{N}=61)$ & $\mathrm{HC}(\mathrm{N}=21)$ & Total $(n=82)$ \\
\hline \multirow[t]{2}{*}{ Colon biopsy } & Procedure completed, N (\%) & $60(98.4)$ & $21(100)$ & $81(98.8)$ \\
\hline & $\begin{array}{l}\text { At least some biopsies } \\
\text { adequate, } \mathrm{N}(\%)\end{array}$ & $58(95.1)$ & $21(100)$ & $79(96.3)$ \\
\hline \multirow[t]{2}{*}{ Skin biopsy } & Procedure completed, N (\%) & $59(96.7)$ & $21(100)$ & $80(97.6)$ \\
\hline & $\begin{array}{l}\text { At least some biopsies } \\
\text { adequate, } \mathrm{N}(\%)\end{array}$ & $59(96.7)$ & $21(100)$ & $80(97.6)$ \\
\hline \multirow[t]{2}{*}{ Submandibular gland biopsy } & Procedure completed, N (\%) & $56(91.8)$ & $20(95.2)$ & $76(92.7)$ \\
\hline & $\begin{array}{l}\text { At least some biopsies } \\
\text { adequate, } \mathrm{N}(\%)\end{array}$ & $42(68.9)$ & $14(66.7)$ & $56(68.3)$ \\
\hline \multirow[t]{3}{*}{ Lumbar Puncture } & Procedure completed, N (\%) & $54(88.5)$ & $21(100)$ & $75(91.5)$ \\
\hline & $\begin{array}{l}\text { CSF sample with hemoglobin } \\
\text { value }<200 \mathrm{ng} / \mathrm{mL}\end{array}$ & $46(75.4)$ & $19(90.5)$ & $65(79.3)$ \\
\hline & $\begin{array}{l}\text { Mean cerebrospinal fluid } \\
\text { volume obtained (SD; range) }\end{array}$ & $15.56(2.3 ; 7.5-21.0)$ & $15.90(1.5 ; 15.0-19.5)$ & $15.66(2.1 ; 7.5-21.0)$ \\
\hline \multirow[t]{2}{*}{ Saliva Collection } & Procedure completed, N (\%) & $57(93.4)$ & $21(100)$ & $78(95.1)$ \\
\hline & $\begin{array}{l}\text { Mean Saliva fluid volume } \\
\text { obtained mL (SD; range) }\end{array}$ & $4.08(2.2 ; 0.40-10.00)$ & $4.92(2.4 ; 0.80-10.00)$ & $4.30(2.2 ; 0.40-10.00)$ \\
\hline Phlebotomy & Procedure completed, $\mathrm{N}(\%)$ & $60(98.4)$ & $21(100)$ & $81(98.8)$ \\
\hline
\end{tabular}

the CRO and stained. Ninety-six (5.4\%) slides were determined to not meet the minimum staining quality required.

\section{DISCUSSION}

Our results demonstrate that multisite tissue and biofluid sampling for $\alpha$-synuclein in a multicenter study is feasible and generally safe. It is notable that it is possible to recruit a cohort to undergo relatively intensive and demanding study procedures with a high procedure completeness rate over just a 22-month study period. The yield of skin and colon biopsies was very high, and was fair for submandibular gland. Additionally, despite the logistical complexity of multiple study sites and cores, nationally and internationally, the number of successfully 
Table 3

Safety of Tissue and Biofluid Collection

\begin{tabular}{|c|c|c|c|c|}
\hline Specimen type & Metric & PD & $\mathrm{HC}$ & Total \\
\hline \multirow[t]{8}{*}{ Colon biopsy } & Procedure completed, $\mathrm{N}$ & 60 & 21 & 81 \\
\hline & Number of subjects with AE from colon biopsy, $\mathrm{N}$ ( $\%$ of those completed) & $0(0)$ & $1(4.8)^{*}$ & $1(1.2)$ \\
\hline & Total number of AE from colon biopsy, $\mathrm{N}$ & 0 & 1 & 1 \\
\hline & Mild (Grade 1) AE from colon biopsy, $\mathrm{N}$ & 0 & 1 & 1 \\
\hline & Moderate (Grade 2) AE from colon biopsy, $\mathrm{N}$ & 0 & 0 & 0 \\
\hline & Severe (Grade 3) AE from colon biopsy, N & 0 & 0 & 0 \\
\hline & Life-threating (Grade 4) AE from colon biopsy, N (serious AE) & 0 & 0 & 0 \\
\hline & AE rate & 0 & 0.05 & 0.01 \\
\hline \multirow[t]{8}{*}{ Skin biopsy } & Procedure completed, $\mathrm{N}$ & 59 & 21 & 80 \\
\hline & Number of subjects with AE from skin biopsy, $\mathrm{N}$ (\% of those completed) & $2(3.4)$ & $5(23.8)$ & $7(8.8)$ \\
\hline & Total number of AE from skin biopsy, $\mathrm{N}$ & 2 & 6 & 8 \\
\hline & Mild (Grade 1) AE from skin biopsy, N & 2 & 6 & 8 \\
\hline & Moderate (Grade 2) AE from skin biopsy, $\mathrm{N}$ & 0 & 0 & 0 \\
\hline & Severe (Grade 3) AE from skin biopsy, N & 0 & 0 & 0 \\
\hline & Life-threating (Grade 4) AE from skin biopsy, N (serious AE) & 0 & 0 & 0 \\
\hline & $\mathrm{AE}$ rate & 0.03 & 0.29 & 0.10 \\
\hline \multirow[t]{8}{*}{ Submandibular gland biopsy } & Procedure completed, $\mathrm{N}$ & 56 & 20 & 76 \\
\hline & Number of subjects with AE from SMG biopsy, N (\% of those completed) & $30(53.6)$ & $13(65.0)$ & $43(56.6)$ \\
\hline & Total number of AE from SMG biopsy, $\mathrm{N}$ & 52 & 18 & 70 \\
\hline & Mild (Grade 1) AE from SMG biopsy, N & 41 & 12 & 53 \\
\hline & Moderate (Grade 2) AE from SMG biopsy, N & 11 & 6 & 17 \\
\hline & Severe (Grade 3) AE from SMG biopsy, N & 0 & 0 & 0 \\
\hline & Life-threating (Grade 4) AE from SMG biopsy, N (serious AE) & 1 & 0 & 1 \\
\hline & $\mathrm{AE}$ rate & 0.93 & 0.90 & 0.92 \\
\hline \multirow[t]{8}{*}{ Lumbar Puncture } & Procedure completed, $\mathrm{N}$ & 54 & 21 & 75 \\
\hline & Number of subjects with AE from CSF acquisition, $\mathrm{N}$ ( $\%$ of those completed) & $8(14.8)$ & $1(4.8)$ & $9(12.0)$ \\
\hline & Total number of AE from CSF acquisition, $\mathrm{N}$ & 10 & 1 & 11 \\
\hline & Mild (Grade 1) AE from CSF acquisition, $N$ & 10 & 1 & 11 \\
\hline & Moderate (Grade 2) AE from CSF acquisition, $\mathrm{N}$ & 0 & 0 & 0 \\
\hline & Severe (Grade III) AE from CSF acquisition, $\mathrm{N}$ & 0 & 0 & 0 \\
\hline & Life-threating (Grade 4) AE from CSF acquisition, N (serious AE) & 0 & 0 & 0 \\
\hline & $\mathrm{AE}$ rate & 0.19 & 0.05 & 0.15 \\
\hline \multirow[t]{2}{*}{ Saliva Collection } & Procedure completed, $\mathrm{N}$ & 57 & 21 & 78 \\
\hline & Number of subjects with $\mathrm{AE}$ from saliva acquisition, $\mathrm{N}$ ( $\%$ of those completed) & 0 & 0 & 0 \\
\hline \multirow[t]{2}{*}{ Phlebotomy } & Procedure completed, N & 60 & 21 & 81 \\
\hline & Number of subjects with AE from phlebotomy, $\mathrm{N}$ & 0 & 0 & 0 \\
\hline
\end{tabular}

*AEs from colon biopsy were assessed for only 80 subjects; there was one HC subject who completed the colon biopsy but documentation of post-procedural AEs assessment was not completed for this subject. AE, adverse event.

stained slides was high. Importantly, there was only one serious adverse event across all procedures.

Generally, procedure completeness, success, and adverse event rate is operator-dependent to a large extent. For each of the procedures conducted, peer training occurred via webinar by a specialist experienced in the procedure of interest. At each study site, there were varying degrees of prior experience with the procedures performed.

A Neurologist or Neurology physician assistant (PA) performed the skin biopsies at several sites. Most Neurologists and Neurology PAs do not have training in skin biopsy, so it is of note that the yield of skin biopsy was high, supporting the feasibility of Neurologists/Neurology PAs to learn this procedure with targeted training and also perform this procedure with high yield. Regarding the SMG biopsy procedure, all otolaryngologists who performed it for S4 had reported previously performing SMG biopsy for clinical purposes, but prior volume and situational experience with it was not captured in study data. Despite the variability that could be introduced by having otolaryngologists at different sites and with different levels of experience performing SMG biopsies, the yield of adequate SMG in this study of $77 \%$ (of attempted) is similar to or greater than what has been reported in other single-site studies. In a study involving SMG biopsy, Adler et al. [2] defined insufficient biopsy as a maximal aggregated area of SMG tissue of $<2 \mathrm{~mm}^{2}$, as was done for S4. Based on that definition, 8 of their 35 subjects (23\%) had insufficient specimens. Given anatomic 
considerations, this lower yield in SMG biopsy compared to colon and skin biopsy is not surprising. The increased risk of inadequate specimen with higher age is also not surprising, as SMG volume decreases with age [12]. Data on whether ultrasound guidance for SMG biopsy significantly improves yield are limited. Some literature suggests increased yield of normal gland and gland tumors with ultrasound guidance [13, 14]. However, one prior study of SMG biopsy for PD/RBD utilized ultrasound but achieved adequate tissue in less than $50 \%$ of subjects [3]. In addition, use of ultrasound was seen as a significant logistical limitation at many S4 sites (both in terms of availability of ultrasound machines and technicians to the otolaryngologists as well as the training required to use ultrasound by the otolaryngologists) and was therefore made optional. In S4, the one site that used ultrasound-guidance for the SMG biopsy had the highest percentage acquisition of adequate tissue but this did not significantly differ from another site that did not use ultrasound guidance.

Collection of all specimens occurred in $83 \%$ of the cohort as a whole; this was higher in the $\mathrm{HC}$ group as compared to the PD group though the difference between the groups did not reach statistical significance. It is possible that completion of some of the procedures was more challenging in the PD group due to their disease manifestations (eg fatigue, mobility issues). Data on specific reasons for lack of completion of specific study activities were not collected in this study and warrant systematic tracking in future studies. As for the adequacy of specimens collected, for the cohort as a whole, all specimens were collected and adequate in $61 \%$. The difference between proportion of subjects with all specimens collected vs. all specimens collected and adequate is driven mainly by the rate of inadequate submandibular gland specimens: in 20 subjects, submandibular gland biopsy was completed but yielded no target tissue. In addition, $10 \mathrm{CSF}$ specimens had $\mathrm{Hb}>200 \mathrm{ng} / \mathrm{mL}$ which may affect measurement of total $\alpha$-synuclein in those samples as well. Higher age was associated with increased odds of an inadequate SMG specimen, and the majority of cases with CSF $\mathrm{Hb}>200 \mathrm{ng} / \mathrm{mL}$ were in the PD group. Otherwise, we did not find predictors of inadequate specimens. However, these analyses are limited by small sample sizes. Thus, all multivariate analyses presented were underpowered to detect differences and should be interpreted with great caution.

While the rate of adverse events related to SMG biopsy was high, the vast majority of AEs were mild.
A similarly high mild AE rate for SMG biopsy in PD has occurred in other studies: 77\% [2]. Reassuringly, the number of serious adverse events in S4 was limited to a single instance and has not been reported in other studies of SMG biopsy in PD or RBD [2, 3, 15]. Increasing the number of passes into the gland with the core needle biopsy tool likely increases both yield and AE rate, and these considerations will both need to be weighed in future studies of SMG biopsy in PD. We did not identify any significant predictors of adverse event occurrence, but again this should be interpreted with caution given the relatively small sample size.

The results presented here give confidence that if, with future analyses of S4 results, $\alpha$-synuclein in any of the obtained tissues and biofluids emerges as a promising PD biomarker, further studies to validate such a biomarker can be feasibly and safely conducted. When taking the feasibility and safety results together and contrasting them across all specimens obtained, SMG biopsy is notable for having the lowest yield and highest adverse event rate. Thus, if S4 demonstrates potential utility of SMG $\alpha$-synuclein as a PD biomarker, future work will be needed to improve yield of SMG biopsy, and improve on the adverse event rate. On the other hand, if measures of $\alpha$-synuclein in other peripheral sites or biofluids demonstrate comparable accuracy to SMG $\alpha$-synuclein as PD biomarkers, the safety profile of SMG biopsy may render SMG $\alpha$-synuclein of lower priority for future development as PD biomarker, in favor of safer alternatives.

We have demonstrated that multicenter tissue and biofluid sampling for $\alpha$-synuclein in PD is feasible and safe. These data will be useful in estimating biopsy yield in sample size calculations for future PD clinical trials and observational studies incorporating tissue sampling. Future analyses of these S4 specimens will address the question of intra-individual distribution of $\alpha$-synuclein in PD.

\section{ACKNOWLEDGMENTS}

This study was funded by the Michael J Fox Foundation.

In-Kind Contribution: Study DaTscan provided by GE Healthcare.

\section{CONFLICT OF INTEREST}

The authors have no conflict of interest to report. 


\section{REFERENCES}

[1] Eriksen JL, Przedborski S, Petrucelli L (2005) Gene dosage and pathogenesis of Parkinson's disease. Trends Mol Med 11, 91-96.

[2] Adler CH, Dugger BN, Hentz JG, Hinni ML, Lott DG, Driver-Dunckley E, Mehta S, Serrano G, Sue LI, Duffy A, Intorcia A, Filon J, Pullen J, Walker DG, Beach TG (2016) Peripheral synucleinopathy in early Parkinson's disease: Submandibular gland needle biopsy findings. Mov Disord 31, 250-256.

[3] Vilas D, Iranzo A, Tolosa E, Aldecoa I, Berenguer J, Vilaseca I, Marti C, Serradell M, Lomena F, Alos L, Gaig C, Santamaria J, Gelpi E (2016) Assessment of alpha-synuclein in submandibular glands of patients with idiopathic rapideye-movement sleep behaviour disorder: A case-control study. Lancet Neurol 15, 708-718.

[4] Lee JM, Derkinderen P, Kordower JH, Freeman R, Munoz DG, Kremer T, Zago W, Hutten SJ, Adler CH, Serrano GE, Beach TG (2017) The search for a peripheral biopsy indicator of alpha-synuclein pathology for Parkinson disease. J Neuropathol Exp Neurol 76, 2-15.

[5] Visanji NP, Mollenhauer B, Beach TG, Adler CH, Coffey CS, Kopil CM, Dave KD, Foroud T, Chahine L, Jennings D, Systemic Synuclein Sampling Study (S4) (2017) The Systemic Synuclein Sampling Study: Toward a biomarker for Parkinson's disease. Biomark Med 11, 359-368.

[6] Seibyl JP, Marek K, Sheff K, Zoghbi S, Baldwin RM, Charney DS, van Dyck CH, Innis RB (1998) Iodine-123beta-CIT and iodine-123-FPCIT SPECT measurement of dopamine transporters in healthy subjects and Parkinson's patients. J Nucl Med 39, 1500-1508.

[7] Systemic Synuclein Sampling Study (S4). (March 2016) S4 Biologics Mannual, https://www.michaeljfox. org/files/S4_Biologics_Manual_Version_2.pdf, March 2016, Accessed August 14, 2018.

[8] Beach TG, Serrano GE, Kremer T'CM, Dziadek S, Sade H, Derkinderen P, Corbille AG, Letournel F, Munoz D, White C, Schneider J, Crary JF, Sue LI, Adler CH, Glass MJ, Intorcia AJ, Walker JE, Foroud T, Coffey CS, Ecklund D, Riss H, Goßmann J, König F, Kopil CM, Arnedo V, Riley L, Linder C, Dave KD, Jennings D, Seibyl J, Mollenhauer B, Chahine LM, and the Systemic Synuclein Sampling Study
(S4) (2018) Immunohistochemical method and histopathology judging for the Systemic Synuclein Sampling Study (S4). J Neuropathol Exp Neurol 77, 793-802.

[9] Goldman JG, Andrews H, Amara A, Naito A, Alcalay RN, Shaw LM, Taylor P, Xie T, Tuite P, Henchcliffe C, Hogarth P, Frank S, Saint-Hilaire MH, Frasier M, Arnedo V, Reimer AN, Sutherland M, Swanson-Fischer C, Gwinn K, Fox Investigation of New Biomarker Discovery, Kang UJ (2018) Cerebrospinal fluid, plasma, and saliva in the BioFIND study: Relationships among biomarkers and Parkinson's disease features. Mov Disord 33, 282-288.

[10] Kang JH, Irwin DJ, Chen-Plotkin AS, Siderowf A, Caspell C, Coffey CS, Waligorska T, Taylor P, Pan S, Frasier M, Marek K, Kieburtz K, Jennings D, Simuni T, Tanner CM, Singleton A, Toga AW, Chowdhury S, Mollenhauer B, Trojanowski JQ, Shaw LM, and the Parkinson's Progression Markers Initiative (2013) Association of cerebrospinal fluid beta-amyloid 1-42, T-tau, P-tau181, and alpha-synuclein levels with clinical features of drug-naive patients with early Parkinson disease. JAMA Neurol 70, 1277-1287.

[11] US Department of Health and Human Services. NCI Common Terminology Criteria for Adverse Events (CTCAE), https://evs.nci.nih.gov/ftp1/CTCAE/About.html, Accessed April 2, 2018.

[12] Kim SK, Allen ED (1994) Structural and functional changes in salivary glands during aging. Microsc Res Tech 28, 243253.

[13] Khan N, Afroz N, Agarwal S, Khan MA, Ahmad I, Ansari HA, Chauhan S, Rabindranath D, Khan AA (2015) Comparison of the efficacy of the palpation versus ultrasonography-guided fine-needle aspiration cytology in the diagnosis of salivary gland lesions. Clin Cancer Investig $J$ 4, 134-139.

[14] Song IH, Song JS, Sung CO, Roh JL, Choi SH, Nam SY, Kim SY, Lee JH, Baek JH, Cho KJ (2015) Accuracy of core needle biopsy versus fine needle aspiration cytology for diagnosing salivary gland tumors. J Pathol Transl Med 49, 136-143.

[15] Adler CH, Dugger BN, Hinni ML, Lott DG, DriverDunckley E, Hidalgo J, Henry-Watson J, Serrano G, Sue LI, Nagel T, Duffy A, Shill HA, Akiyama H, Walker DG, Beach TG (2014) Submandibular gland needle biopsy for the diagnosis of Parkinson disease. Neurology 82, 858-864. 


\section{APPENDIX}

SYSTEMIC SYNUCLEIN SAMPLING STUDY (S4) ADDITIONAL AUTHORS

Lindsey Guilmette ${ }^{1}$, David Russell ${ }^{1}$, Chaucer Noyes-Lloyd ${ }^{1}$, Colleen Mitchell ${ }^{2}$, Danielle Smith ${ }^{2}$, Madeline Potter ${ }^{2}$, Rose Case ${ }^{2}$, David Lott ${ }^{3}$, Amy Duffy $^{3}$, Penelope Hogarth ${ }^{4}$, Madeline Cresswell ${ }^{4}$, Rizwan Akhtar ${ }^{5}$, Rachael Purri ${ }^{5}$, Amy Amara ${ }^{6}$, Courtney Blair ${ }^{6}$, Ali Keshavarzian ${ }^{7}$, Connie Marras ${ }^{8}$, Naomi Visanji ${ }^{8}$, Brandon Rothberg ${ }^{8}$, Vikash $\mathrm{Oza}^{9}$
${ }^{1}$ Institute for Neurodegenerative Disorders, New Haven, CT, USA

${ }^{2}$ Indiana University, Indianapolis, IN, USA

${ }^{3}$ Mayo Clinic, Phoenix, AZ, USA

${ }^{4}$ Oregon Health and Science University, Portland, OR, USA

${ }^{5}$ University of Pennsylvania, Philadelphia, PA, USA

${ }^{6}$ University of Alabama at Birmingham, Birmingham, AL, USA

${ }^{7}$ Rush University, Chicago, IL, USA

${ }^{8}$ University Health Network, Toronto, Canada

${ }^{9}$ New York University, New York, NY, USA 